\title{
Bilateral aggressive malignant granulosa cell tumour with essentially different immunophenotypes in primary and metastatic lesions comprising predominantly sarcomatoid and fibrothecomatous patterns - looking for prognostic markers: a case report
}

Wojciech Jozwicki ${ }^{1}$, Anna Aneta Brożyna ${ }^{1}$, Małgorzata Walentowicz'르. Marek Grabiec ${ }^{2}$

1Department of Tumour Pathology and Pathomorphology, The Ludwik Rydygier Collegium Medicum of Nicolaus Copernicus University, The Franciszek Lukaszczyk Oncology Centre, Bydgoszcz, Poland

2Oncological Gynaecology and Gynaecological Nursing, The Ludwik Rydygier Collegium Medicum of Nicolaus Copernicus University, The Franciszek Lukaszczyk Oncology Centre, Bydgoszcz, Poland

Submitted: 4 August 2010

Accepted: 3 October 2010

Arch Med Sci 2011; 7, 5: 918-922

DOI: 10.5114 /aoms.2011.25573

Copyright () 2011 \& Termedia

\section{Abstract}

We present an unusual case of a young woman with rare bilateral, very aggressive ovarian granulosa cell tumour (GCT), comprised of granulosa, sarcomatoid and fibrothecomatous fields with significantly different immunostaining of primary and metastatic tumours showing stronger WT1, Bcl2, fascin and EGFR expression in metastases. Despite radical surgery and chemotherapy the tumour recurred rapidly and the patient died 16 months later. Such results clearly demonstrate the usefulness of immunostaining for the above markers as prognostic/predictive factors and the need for careful assessment of the immunoprofile of both primary and metastatic tumours, which can be useful for therapy and follow-up planning in GCT cases.

Key words: immunoprofile, sarcomatoid differentiation, fibrothecomatous differentiation.

\section{Introduction}

Granulosa cell tumours (GCTs) are rare tumours, accounting for $2-5 \%$ of primary ovarian tumours, that develop unilaterally (>95\% of cases) in peri- and postmenopausal women. The tumour behaviour is difficult to predict. Little information about definitive prognostic markers, except for the stage, has been reported [1]. These tumours have malignant potential, although metastasis or recurrence usually occurs late, as much as 20-30 years following the primary diagnosis [2].

Here we report an unusual case of a young woman who developed bilateral ovarian GCTs with a very aggressive course, rapid recurrence, and a short time of survival. We examined the immunostaining pattern of a broad range of markers, trying to identify indicators of this phenotype of GCT.

\author{
Corresponding author: \\ Wojciech Jozwicki MD, PhD \\ Department of Tumour \\ Pathology \\ and Pathomorphology \\ The Franciszek Lukaszczyk \\ Oncology Centre \\ The Ludwik Rydygier \\ Collegium Medicum \\ of Nicolaus Copernicus \\ University \\ 2 Izabela Romanowska Str \\ 85-796 Bydgoszcz, Poland \\ Phone/fax: +48 0523743343 \\ E-mail: \\ wojtekj@cm.umk.bydgoszcz.pl, \\ jozwickiw@co.bydgoszcz.pl
}




\section{Case report}

A 35-year-old nulliparous woman was admitted to the Department of Gynaecological Oncology, Oncology Centre, Bydgoszcz, Poland, for a relaparotomy. Three months earlier, she underwent a laparotomy. During the surgical procedure, an encapsulated tumour $30 \mathrm{~cm}$ in diameter, filling the small pelvis, in adhesion with the sigmoid colon and lesser omentum, was found. The tumour was enucleated. The intraoperative pathological examination results were ambiguous and revealed only the presence of atypical cells. Due to young age, nulliparous status and imprecise histopathological diagnosis, the decision concerning possible radical surgery was postponed until an unequivocal diagnosis could be obtained. The postoperative material was consulted at the Maria SkłodowskaCurie Memorial Cancer Centre and Institute of Oncology, Warsaw, Poland and the malignant lesion was diagnosed. Before the second laparotomy, ultrasonography revealed only a solid cystic mass in the left ovary. Tumour markers were normal (AFP $3.11 \mathrm{lU} / \mathrm{ml}$, CA-125 $19.51 \mathrm{U} / \mathrm{ml}$ and CEA $0.4 \mathrm{U} / \mathrm{ml}$ ). At the re-laparotomy, a left ovarian tumour $7 \mathrm{~cm}$ in diameter and an $8 \mathrm{~cm}$ tumour of the small intestine adherent to the sigmoid and left appendages were discovered. The patient underwent a radical hysterectomy, and tumour masses in the mesentery of the small intestine and in the colon were removed with clear margins. The tumour comprised mainly atypical fibrothecomatous and sarcomatoid patterns, with a trace of granulosa cell differentiation surrounded by spindle-shaped stromal cells. The mesenteric and colonic tumours comprised sarcomatoid and sarcomatoid/fibrothecomatous components, respectively. Histochemistry and immunocytochemistry indicated GCT (Figure 1). The tumours comprised more than 60\% Ki67-positive cells and numerous cells with bizarre nuclei. The immunophenotypes of the ovarian, mesenteric, and colonic tumours differed (Table I, Figure 2). Immunostaining for CD56, CD99, and calretinin was negative in the sarcomatoid areas, and their expression levels were lower in fields with mixed fibrothecomatous and sarcomatoid differentiations than in fibrothecomatous regions of the tumour. Moreover, the metastatic cells showed stronger and more extensive staining for CD117 and CK AE1/3 (both components), and weaker or no staining for calretinin and CK MNF116. Both the ovarian and metastatic tumours were negative for
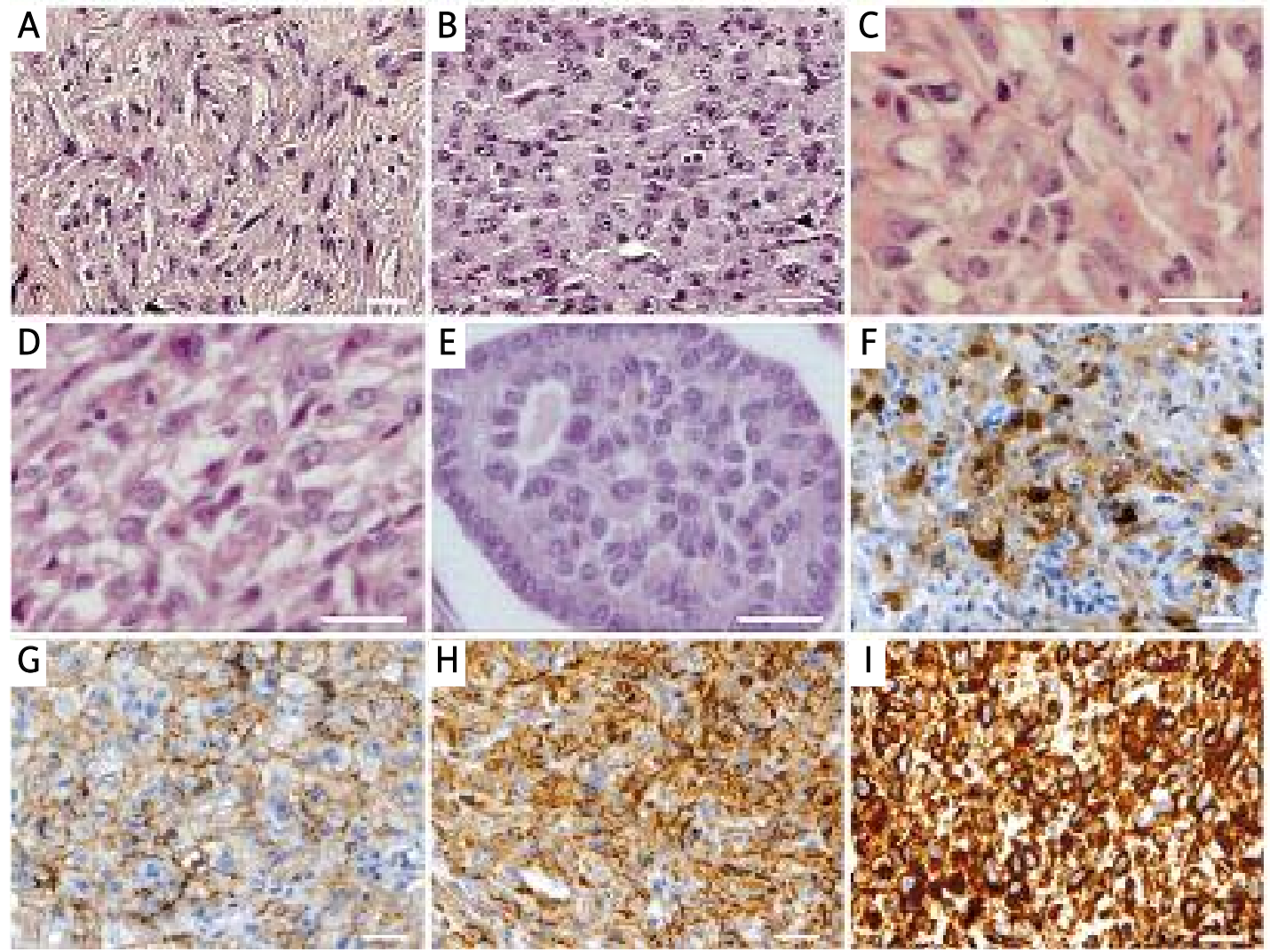

Figure 1. Histopathological and immunohistochemical features of malignant granulosa cell tumour. The tumour was composed predominantly of sarcomatoid (A) and thecomatous (B-D) cells with vacuolated cytoplasm. A trace of Call-Exner bodies differentiation type was also seen (E). Tumour cells stained positively for calretinin (F), CD56 (G), CD99 $(H)$, and vimentin (I). Immunoreactivity was visualized with DAB, and the sections were counterstained with haematoxylin. Scale bars $=50 \mu \mathrm{m}$ 
Table I. Expression of selected markers of the primary and metastatic (mesenteric and colonic) tumours

\begin{tabular}{|c|c|c|}
\hline Marker & Tumour & $\begin{array}{c}\text { Immunostaining } \\
\text { (intensity/\% } \\
\text { of area; } \\
\text { localization) }\end{array}$ \\
\hline \multirow[t]{4}{*}{ EGFR } & Primary & - \\
\hline & Mesenteric & - \\
\hline & Colonic: sarcomatoid field & - \\
\hline & Colonic: thecomatous field & $+/ 75 ; C$ \\
\hline \multirow[t]{4}{*}{ WT1 } & Primary & $+/ 10 ; C$ \\
\hline & Mesentery & $+/ 5$; C \\
\hline & Colonic: sarcomatoid field & $++/ 30 ;+++/ 20 ; C$ \\
\hline & Colonic: thecomatous field & - \\
\hline \multirow[t]{4}{*}{$\mathrm{BCl} 2$} & Primary & $+/ 1$; C \\
\hline & Mesentery & - \\
\hline & Colonic: sarcomatoid field & $++/ 20 ; C$ \\
\hline & Colonic: thecomatous field & - \\
\hline \multirow[t]{4}{*}{$E R \beta$} & Primary & $+/ 70 ; C, N$ \\
\hline & Mesentery & $+/ 35 ;++/ 50 ; C, N$ \\
\hline & Colonic: sarcomatoid field & $++/ 70 ; \mathrm{C}, \mathrm{N}$ \\
\hline & Colonic: thecomatous field & $++/ 100 ; C, N$ \\
\hline \multirow[t]{4}{*}{ Fascin } & Primary & $+++/ 90 ; C$ \\
\hline & Mesentery & $++/ 45 ;+++/ 40 ; C$ \\
\hline & Colonic: sarcomatoid field & $\begin{array}{c}+/ 10 ;++/ 50 \\
+++/ 30 ; C\end{array}$ \\
\hline & Colonic: thecomatous field & $+/ 50$; C \\
\hline
\end{tabular}

$\mathrm{C}$ - cytoplasmic staining, $\mathrm{N}$ - nuclear staining. Staining intensity was scored as follows: negative - , weak + , moderate ++ , and strong +++

p73, p63, p53, CA125, desmin, CD34, E-cadherin, CK5/6, CK20, and CK HMW. Although HER2 immunostaining was negative, chromogenic in situ hybridization revealed an increased number of HER2 gene copies assessed according to a breast cancer evaluation protocol (Zymed Inc.) up to 4.4 and 2.1 copies per bizarre and non-bizarre nuclei, respectively.

Ten days after surgery, positron emission tomography-computed tomography revealed no abnormal fluorodeoxyglucose uptake. The patient received bleomycin, etoposide, and cisplatin combination chemotherapy (3 courses, total dose of bleomycin was $1800 \mathrm{mg}$ (9 administrations), etoposide $-210 \mathrm{mg}$ (7 administrations) and cisplatin - $495 \mathrm{mg}$ (9 administrations)). Systematic follow-up examination was performed every 2 months. Lung $X$-ray and NMR (nuclear magnetic resonance) imaging at 6 months and abdominal cavity ultrasound examinations 6 and 8 months after the re-laparotomy revealed no atypical findings. The serum levels of tumour markers after the re-laparotomy (examined 6 times) were normal (CA-125 from $12.74 \mathrm{U} / \mathrm{ml}$ to $15.72 \mathrm{U} / \mathrm{ml}$; $\mathrm{LDH}$ from $221 \mathrm{U} / \mathrm{l}$ to $395 \mathrm{U} / \mathrm{l}$, AFP from $3.92 \mathrm{ng} / \mathrm{ml}$ to $7.01 \mathrm{ng} / \mathrm{ml}$, HCG from $0 \mathrm{mlU} / \mathrm{ml}$ to $0.73 \mathrm{mlU} / \mathrm{ml}$, CEA from $0.8 \mathrm{ng} / \mathrm{ml}$ to $1.1 \mathrm{ng} / \mathrm{ml}$ and Ca $15-3$ from $11.9 \mathrm{U} / \mathrm{ml}$ to $13.3 \mathrm{U} / \mathrm{ml}$ ). Tumour recurrence ensued after a 14-month asymptomatic course, and a lesion measuring $6 \mathrm{~cm} \times 5.3 \mathrm{~cm} \times 12.0 \mathrm{~cm}$ infiltrating the bladder wall was diagnosed with NMR imaging. The patient received palliative radiotherapy (20 Gy in five fractions of 400 cGy; Co-60 radiation/6 MV/15 MV). The patient died 16 months after the re-laparotomy.

\section{Discussion}

The dynamics of the carcinogenesis process, which affect the biological malignancy of the tumour, are regulated by various genetic and epigenetic factors, including microenvironment and status and condition of the immune system $[2,3]$. Tissue changes during the menstrual cycle or pregnancy can reflect epigenetic and microenvironmental hormonal regulation, which can also affect the development and progression of the tumour [2-4]. Epithelial cancers, arising in women mostly within organs related to reproduction (ovary, breast, cervix), seem to be highly sensitive to hormonal changes, particularly in younger patients.

The GCTs metastasize and recur only in $20 \%$ of patients 10 years after the original diagnosis. However, in our patient the tumour recurred very rapidly, despite radical surgery and chemotherapy. The stage at the time of diagnosis is the most important prognostic factor of GCTs. It is very important to identify new markers that could help to predict aggressive course, rapid recurrence or chemotherapy response. There are only a few immunohistochemical prognostic markers in GCTs $[1,5]$. One of our purposes was to determine the immunostaining profile of an ovarian metastatic GCT and indicate potential prognostic markers.

The tumour of our patient showed the expression of sex cord-stromal marker (calretinin) and a very high mitotic index (28/10 HPF) compared with most patients with a GCT $(<5 / 10 \mathrm{HPF})[2,5]$. Unlike McCluggage et al. [6], who reported on a malignant fibrothecomatous tumour belonging to the same group of sex cord-stromal tumours as GCTs, we observed different immunophenotypes in the primary and metastatic neoplasms. We found very clear expression of WT1, in contrast to Cathro and Stoler [7], who found weak or variable WT1 expression. High expression of WT1 can be related to an unfavourable prognosis in tumour patients [8]. In vitro studies show that cell lines expressing WT1 and Bcl 2 are resistant to chemotherapeutic agents [9]. In our patient, WT1-induced upregulation of antiapoptotic Bcl2 protein might have caused chemoresistance of the metastatic sarcomatoid 

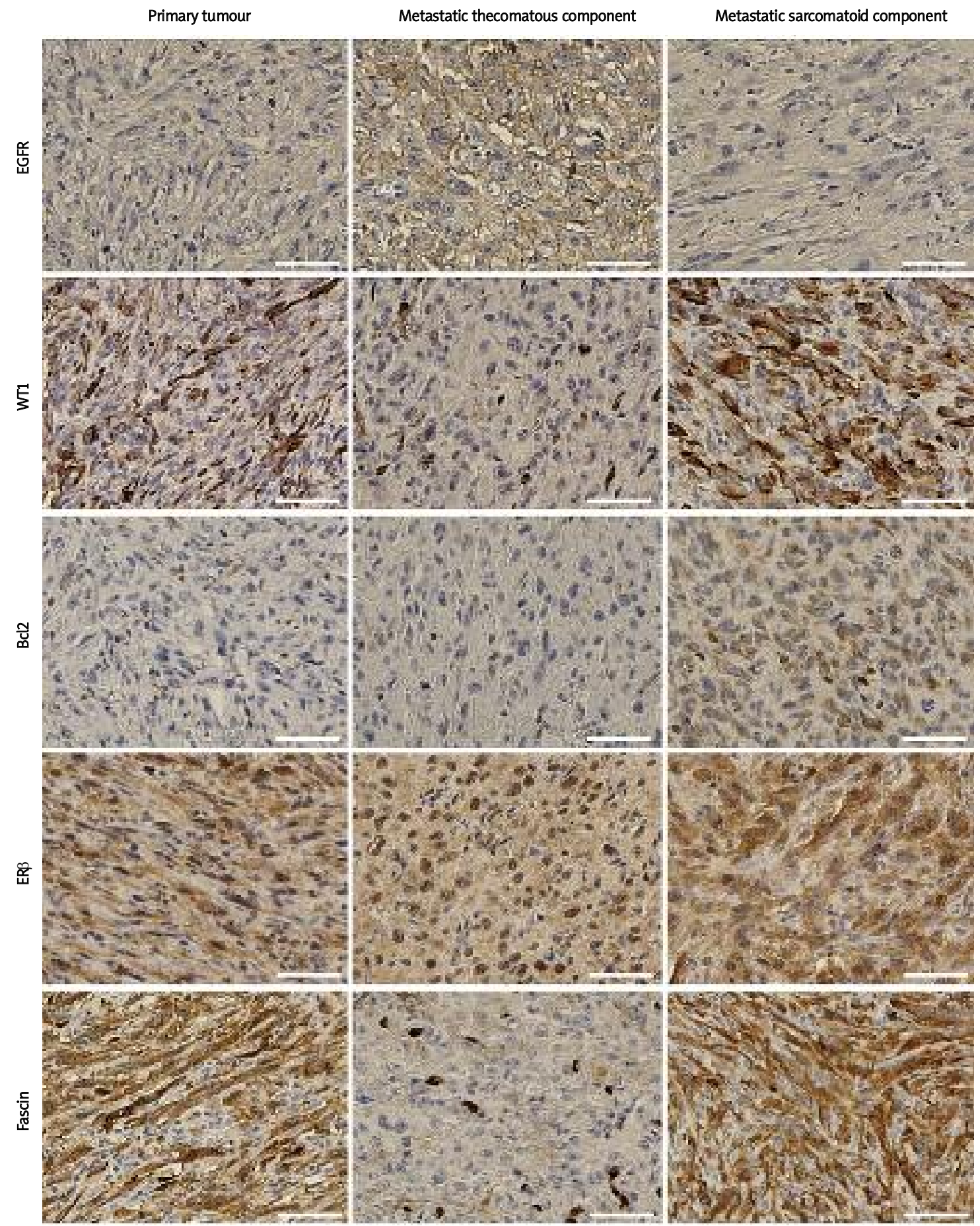

Figure 2. Malignant granulosa cell tumour showing different immunophenotype of the primary and metastatic lesions. Immunoreactivity was visualized with DAB, and the sections were counterstained with haematoxylin. Scale bars $=100 \mu \mathrm{m}$

cells. Similarly, chemoresistance of fibrothecomatous cells and more aggressive course might have resulted from overexpression of EGFR [10, 11]. Weaker and less abundant expression of fascin within the metastatic tumours might indicate a more favourable prognosis [12], although in our patient, its expression was as strong within the sarcomatoid component as in the primary tumour.
In our patient, the greater expression of EGFR, $\mathrm{BCl}-2$, and WT1 in the metastatic lesions and fascin in the primary tumour seemed to be a predictor of the poor course of GCT and to reflect a more aggressive phenotype, which might indicate early recurrence.

In conclusion, our findings indicate the possible existence of a fundamental difference between 
primary and metastatic cells. These results also indicate the need for more aggressive therapy of GCTs with stronger WT1, Bcl2, fascin, and EGFR expression in the metastases. Immunostaining for the above markers may provide potential predictors of GCT malignancy. We cannot exclude the possibility that metastatic expression of ER $\beta$ and EGFR might suggest the usefulness of hormone and anti-EGFR therapy, respectively.

\section{Acknowledgments}

This study was supported by the Collegium Medicum of Nicolaus Copernicus University and Oncology Centre, Bydgoszcz, Poland. The authors declare that they have no competing interests. Written informed consent was obtained from the patient's mother for publication of this case report and any accompanying images. A copy of the written consent is available for review by the Editorin-Chief of this journal.

\section{References}

1. Aboud E. A review of granulosa cell tumours and thecomas of the ovary. Arch Gynecol Obstet 1997; 259: 161-5.

2. Sex cord-stromal tumors of the ovary. Deavers MT, Oliva E, Nucci MR (eds). Elsevier Churchill Livingstone 2009.

3. Hosseini M, Houshmand M, Ebrahimi A. Breast cancer risk not only was not associated with CYP17/A2 allele but also was related to A1 allele. Arch Med Sci 2009; 5: 103-6.

4. Jakubik J, Gottwald L, Kukulska M, et al. Breast cancer in pregnant women: report of two cases and review of the literature. Arch Med Sci 2008; 4: 204-7.

5. Young RH. Sex cord-stromal tumors of the ovary and testis: their similarities and differences with consideration of selected problems. Mod Pathol 2005; 18 Suppl 2: S81-98.

6. McCluggage WG, Sloan JM, Boyle DD, Toner PG. Malignant fibrothecomatous tumour of the ovary: diagnostic value of anti-inhibin immunostaining. J Clin Pathol 1998; 51: 868-71.

7. Cathro HP, Stoler MH. The utility of calretinin, inhibin, and WT1 immunohistochemical staining in the differential diagnosis of ovarian tumors. Hum Pathol 2005; 36: 195-201.

8. Miyoshi Y, Ando A, Egawa C, et al. High expression of Wilms' tumor suppressor gene predicts poor prognosis in breast cancer patients. Clin Cancer Res 2002; 8: 1167-71.

9. Mayo MW, Wang CY, Drouin SS, et al. WT1 modulates apoptosis by transcriptionally upregulating the bcl-2 proto-oncogene. Embo J 1999; 18: 3990-4003.

10. Leibl S, Bodo K, Gogg-Kammerer M, et al. Ovarian granulosa cell tumors frequently express EGFR (Her-1), Her-3, and Her-4: an immunohistochemical study. Gynecol Oncol 2006; 101: 18-23.

11. Mendelsohn J, Baselga J. Status of epidermal growth factor receptor antagonists in the biology and treatment of cancer. J Clin Oncol 2003; 21: 2787-99.

12. Wen YH, Yee H, Goswami S, PS Shukla. Fascin expression in serous tumors of ovary correlates with aggressiveness of malignancy. Int J Gynecol Pathol 2009; 28: 187-92. 